\title{
ARTíCULO
}

\section{Morfología de la cavidad visceral del pez jorobado Selene vomer (Teleostei: Carangidae)}

\author{
Morphology of the visceral cavity of the lookdown Selene vomer (Teleostei: \\ Carangidae)
}

\author{
Abraham Kobelkowsky $^{1^{*}}$ y Omar Beltrán-Vinalay ${ }^{2}$ \\ ${ }^{1}$ Laboratorio de Peces, Departamento de Biología, Universidad Autónoma Metropolitana, Unidad Iztapalapa, Av. San Rafael Atlixco \\ 186, col. Vicentina, Iztapalapa, 09340 Ciudad de México, México \\ ${ }^{2}$ Doctorado en Ciencias Biológicas y de la Salud, Universidad Autónoma Metropolitana, Ciudad de México, México \\ *Autor corresponsal: akobelkowsky@gmail.com
}

\begin{abstract}
The lookdown Selene vomer is a teleost representative of the Carangidae family with a distribution in the coasts of the Western Atlantic, characterized by an extreme lateral flattening of his body. There are few records of the anatomy of Selene Lacépéde, 1802. The objective was to determine the relationship between the compressed shape of the body of S. vomer and the organization of the organs within the visceral cavity. By means of a dissection, the skeletal boundaries of the visceral cavity were exposed, and the anatomy of the organs contained in it were analyzed, described, and illustrated. The visceral cavity is laterally flattened, with a trapezoidal lateral aspect, vertical, and extraviscerally extended by means of the secondary visceral cavities. The gas bladder is wide and formed by a single chamber, and it develops two posterior lobes towards the back. The mesonephros is compressed and curved, and it develops two anterior renal lobes. The digestive tube is arranged vertically and shows a set of branched duodenal caeca. The gonads have a diagonal position. A direct relationship between the lateral flattening of the body with the compressed shape of the visceral cavity and its organs is recognized. The shape of the visceral cavity is related to the vertical lengthening of the scapular and pelvic girdles, the pleural ribs, and the first anal pterygiophore. The organ with the highest number of special anatomical features is the gas bladder, due to its extravisceral posterior lobes and its attachment to the vertebral column by means of ligaments.
\end{abstract}

Key words: Splanchnology, secondary visceral cavities, duodenal caeca, Moonfish, gas bladder

Resumen.- El pez luna o jorobado Selene vomer es un teleósteo representante de la familia Carangidae con distribución en las costas del Atlántico Occidental, caracterizado por el extremo aplanamiento lateral de su cuerpo. Existen escasos registros de la anatomía de Selene Lacépède, 1802. El objetivo fue la determinación de la relación entre la forma comprimida del cuerpo de $S$. vomer y la organización de los órganos dentro la cavidad visceral. Mediante disección se determinaron los límites esqueléticos de la cavidad visceral y se analizó, describió e ilustró la anatomía de los órganos contenidos en la misma. La cavidad visceral es aplanada lateralmente, de aspecto lateral trapezoidal, vertical y extendida extra visceralmente mediante las cavidades viscerales secundarias. La vejiga gaseosa es amplia, unicameral, y desarrolla hacia atrás dos lóbulos posteriores. El mesonefros es comprimido y curvado y forma dos lóbulos renales anteriores. El tubo digestivo se dispone verticalmente y muestra un conjunto de ciegos duodenales ramificados. Las gónadas tienen una posición diagonal. Se reconoce una relación directa del aplanamiento lateral del cuerpo con la forma comprimida de la cavidad visceral y de sus órganos. La forma de la cavidad visceral está relacionada con el alargamiento vertical de las cinturas escapular y pélvica, las costillas pleurales y el primer pterigióforo anal. El órgano con mayor número de rasgos anatómicos especiales es la vejiga gaseosa, por sus lóbulos posteriores extraviscerales y por su anclaje a la columna vertebral mediante ligamentos.

Palabras clave: Esplacnología, cavidades viscerales secundarias, ciegos duodenales, pez luna, vejiga gaseosa

\section{INTRODUCCIÓN}

La distribución de los órganos en la cavidad visceral de los teleósteos, con excepción del bazo, presenta un patrón general, consistente en la ubicación en orden descendente del mesonefros, la vejiga gaseosa o natatoria, el tubo digestivo con sus glándulas anexas y las gónadas. Sin embargo, el conocimiento de las variaciones anatómicas al patrón general de estos órganos, puede ser útil en estudios taxonómicos como los que proporcionan Ogawa (1961) sobre los riñones, Whitehead \& Blaxter (1989) sobre la vejiga gaseosa de clupeidos, Wilson \& Castro (2011) sobre el tubo digestivo de teleósteos, Sasaki (1989) sobre la organografía de Sciaenidae, Collette \& Chao (1975) sobre la organografía de los "bonitos" (Scombridae), Harder (1958), sobre el intestino de engráulidos y Kobelkowsky (2012) sobre los ovarios. 
Asimismo, el conocimiento de los caracteres anatómicos de las vísceras es básico en su interpretación funcional y por lo tanto en la biología de las especies.

De acuerdo con Nelson et al. (2016) el orden Carangiformes incluye seis familias entre las cuales está Carangidae, formada por 30 géneros, entre los que se encuentra Selene Lacepède, 1802. Dentro de la ictiofauna mexicana Selene está representado por las especies del Pacífico: S. brevoortii (Gill, 1863), S. orstedii Lütken, 1880 y $S$. peruviana (Guichenot, 1866), mientras que en el Atlántico por: S. setapinnis (Mitchell, 1815), $S$. brownii (Cuvier, 1816), S. spixii (Castelnaud, 1855) y $S$. vomer (Linnaeus, 1758). La distribución de $S$. vomer es desde Nueva Escocia, Canadá hasta Uruguay, incluyendo Bermudas, Antillas y Golfo de México (Robins \& Ray 1986). Frecuencias esporádicas de la especie han sido registradas en Argentina (Ruocco et al. 2008).

Los peces de la familia Carangidae muestran una cierta diversidad de formas y de grados de aplanamiento lateral, que puede reflejarse en la disposición y morfología de sus órganos internos. S. vomer presenta el cuerpo notablemente comprimido, corto y alto. Como antecedentes sobre la anatomía de los carángidos en México se encuentra el estudio de Guzmán \& Polaco (2002) sobre la hiperostosis de peces mexicanos, con mención de Carangoides otrynter, Caranx caninus, Selene brevoortii, Selene vomer y Trachinotus kennedyi.

Considerando que los peces de la familia Carangidae muestran una cierta diversidad de formas y de grados de aplanamiento lateral del cuerpo, y por lo tanto de sus cavidades celómicas, se espera que la morfología de sus órganos en un representante del género Selene, el cual tiene el cuerpo notablemente comprimido, corto y alto, tenga una relación directa con dichos aspectos morfológicos, por lo cual, el objetivo del presente trabajo fue el análisis, descripción e ilustración de la cavidad visceral y de los órganos que contiene $S$. vomer y determinar la relación entre la forma del cuerpo y organización de sus órganos en dicha cavidad.

\section{MATERIALES Y MÉTODOS}

Se analizaron 10 ejemplares de adultos machos y hembras de Selene vomer, (IBUNAM-PE 626, IBUNAM-PE 4851, IBUNAM-PE 3219, IBUNAM-PE 504, IBUNAM-PE 21814), con un intervalo de tallas de 75,0-170,0 mm, colectados mediante redes de arrastre en lagunas costeras del Estado de Veracruz y litorales del Golfo de México, México, los cuales se fijaron en formaldehido al $10 \%$ y se conservaron en alcohol etílico al 70\%.
Con el objeto de exponer, describir e ilustrar los límites esqueléticos de la cavidad visceral, se retiraron la piel y los miotomos del costado izquierdo del cuerpo en un área rectangular (Fig. 1), hasta evidenciar la serie opercular, las cinturas escapular y pélvica, las costillas pleurales y epipleurales, las vértebras precaudales, las primeras espinas hemales y los primeros pterigióforos anales. Asimismo, con el propósito de observar en sección transversal la cavidad visceral, se realizó un corte transversal del cuerpo del pez al nivel del inicio de la aleta dorsal (Fig. 1, transecto 2a2a'). Con el fin de completar la exposición de la cavidad visceral y de la parte anterior, tanto del mesonefros como del sistema digestivo, se extrajeron los huesos opercular y preopercular, y se desprendieron los filamentos branquiales de los arcos branquiales.

Se analizaron, describieron e ilustraron in situ, el sistema urogenital, la vejiga gaseosa, el tubo digestivo, el hígado, el páncreas y el bazo.

A continuación, se removieron la cintura escapular con su musculatura y las costillas, con el objeto de exponer, analizar e ilustrar los órganos de la cavidad visceral. Se midió la longitud del intestino y se determinó el índice intestinal, relacionando la longitud intestinal con la longitud patrón del pez. Se contaron los ciegos pilóricos o duodenales.

Se realizó una comparación de la forma de la cavidad celómica de $S$. vomer con la de $S$. setapinnis (IBUNAM- PE 7731, IBUNAM-PE 6560) y S. spixii (IBUNAM-PE 7367). Asimismo, se comparó la forma de la cavidad visceral en una serie de especies de Carangidae, que se inicia con una especie de cuerpo hidrodinámico y que termina con otra de cuerpo de gran longitud y baja altura. Para esto se seleccionaron y ordenaron las siguientes especies: Decapterus punctatus (Cuvier, 1829), Oligoplites saurus (Bloch \& Schneider, 1801), Caranx latus (Agassiz, 1831), Hemicaranx amblyrhynchus (Cuvier, 1833), Chloroscombrus chrysurus (Linnaeus, 1766) y Selene vomer.

Se realizaron las ilustraciones mediante un tubo de dibujo (camera lucida) acoplado a un microscopio estereoscópico Leica ${ }^{\circledR}$ Wild M3Z.

La terminología del endoesqueleto siguió el criterio de Gregory (1959) y la de la musculatura siguió el de Winterbottom (1974). 


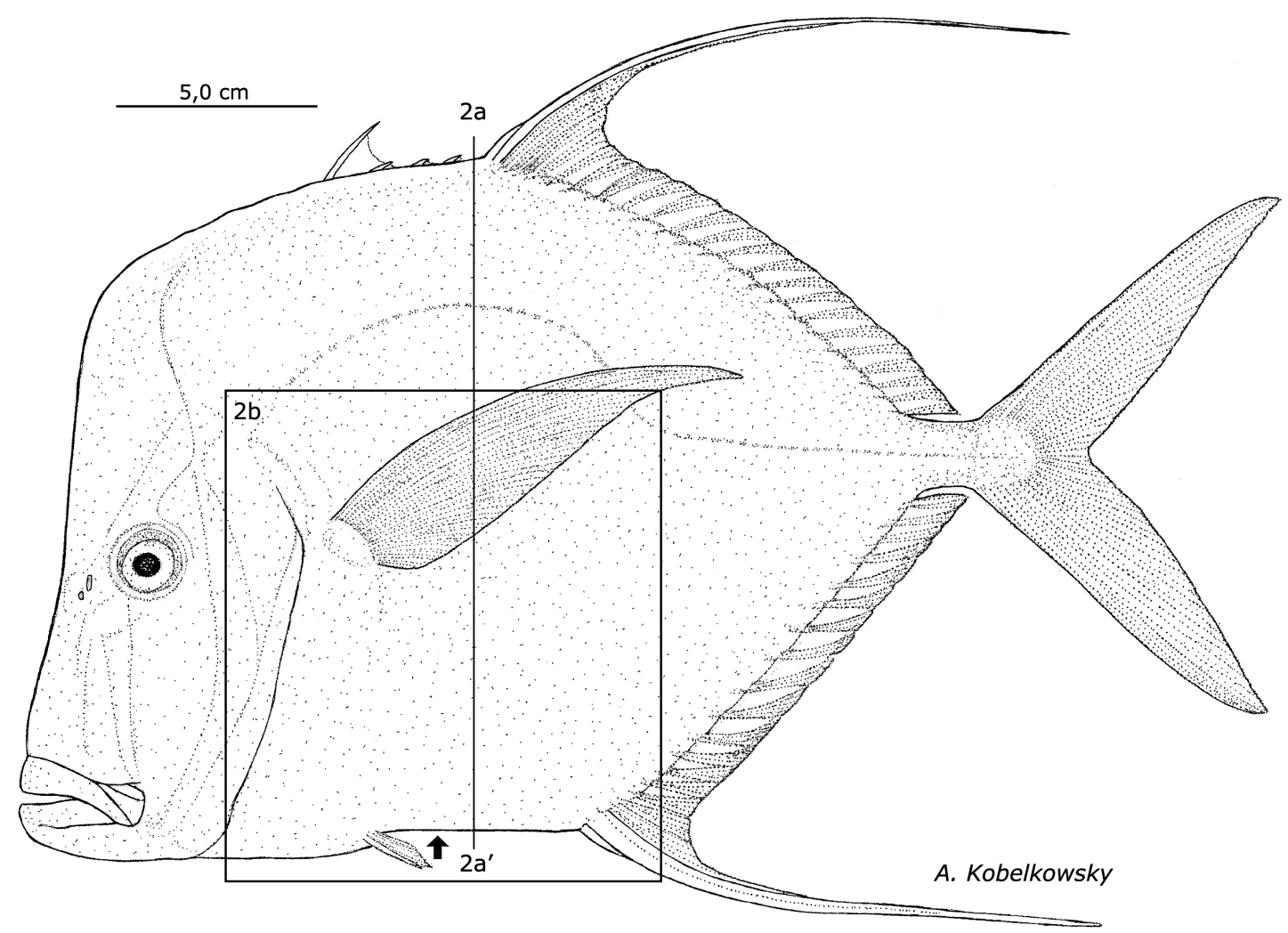

Figura 1. Morfología externa de Selene vomer. $2 a$ y $2 a^{\prime}$ indica el transecto del área representada en la Figura 2a; el recuadro indica el área de lo representado en la Figura $\mathbf{2 b}$. La flecha indica la posición del ano / External morphology of Selene vomer. 2a y 2a' indicates the transect of the area represented in Figure 2a; the box indicates the area of what is represented in Figure $2 \mathrm{~b}$. The arrow points the position of anus

\section{RESUltados}

La altura máxima del cuerpo de $S$. vomer corresponde aproximadamente al 1,42 de la longitud patrón del pez. La posición del ano es cercana a las aletas pélvicas (Fig. 1).

\section{CAVIDAD Visceral}

La cavidad visceral de $S$. vomer es aplanada en el sentido lateral (Fig. 2a); en vista lateral es trapezoidal, con su eje más largo en el sentido vertical (Fig. 3b) y con el extremo posterior bifurcado desde el nivel del complejo "primera espina hemal/primer pterigióforo anal" (Fig. 3a) en las "cavidades viscerales secundarias". Se encuentra delimitada por las 10 vértebras precaudales, las costillas pleurales (ventrales), las cinturas escapular y pélvica, el primer pterigióforo anal, la primera espina hemal (Fig. 2b), los músculos retractores dorsales, la musculatura apendicular, y la musculatura axial.
Las vértebras 7 a 10 muestran sus procesos transversos triangulares y orientados hacia abajo (Fig. 2b); forman puentes hemales en los elementos 8 a 10, por los cuales, pasan tanto la aorta dorsal, como la vena caudal. En ambos lados se localizan las siete costillas pleurales, las que se articulan a las vértebras 3 a 9 (Fig. 2b) y son extraordinariamente largas, con excepción de la última.

El primer pterigióforo anal es notablemente largo (Fig. 2b), siendo su longitud de cerca del $80 \%$ de la altura de la cavidad visceral; es curvado y de posición oblicua y adelantada, con el extremo inferior con forma de pala; se yuxtapone a la primera espina hemal, la cual es relativamente larga, formándose el complejo "primera espina anal/primer pterigióforo anal".

Ambas cinturas (Figs. 2b y 3a) son alargadas en el sentido vertical; la cintura escapular muestra notablemente largos los huesos cleitrales y los coracoides, los cuales no se conectan entre si directamente por su extremo inferior, sino mediante el cartílago escapular (Figs. 2b y 3a); la cintura pélvica es, asimismo, notablemente larga, delgada y de posición oblicua. La musculatura axial que contribuye a los límites laterales de la cavidad visceral se inserta principalmente en las costillas pleurales, siendo su componente principal el hypaxialis (Fig. 2a). 


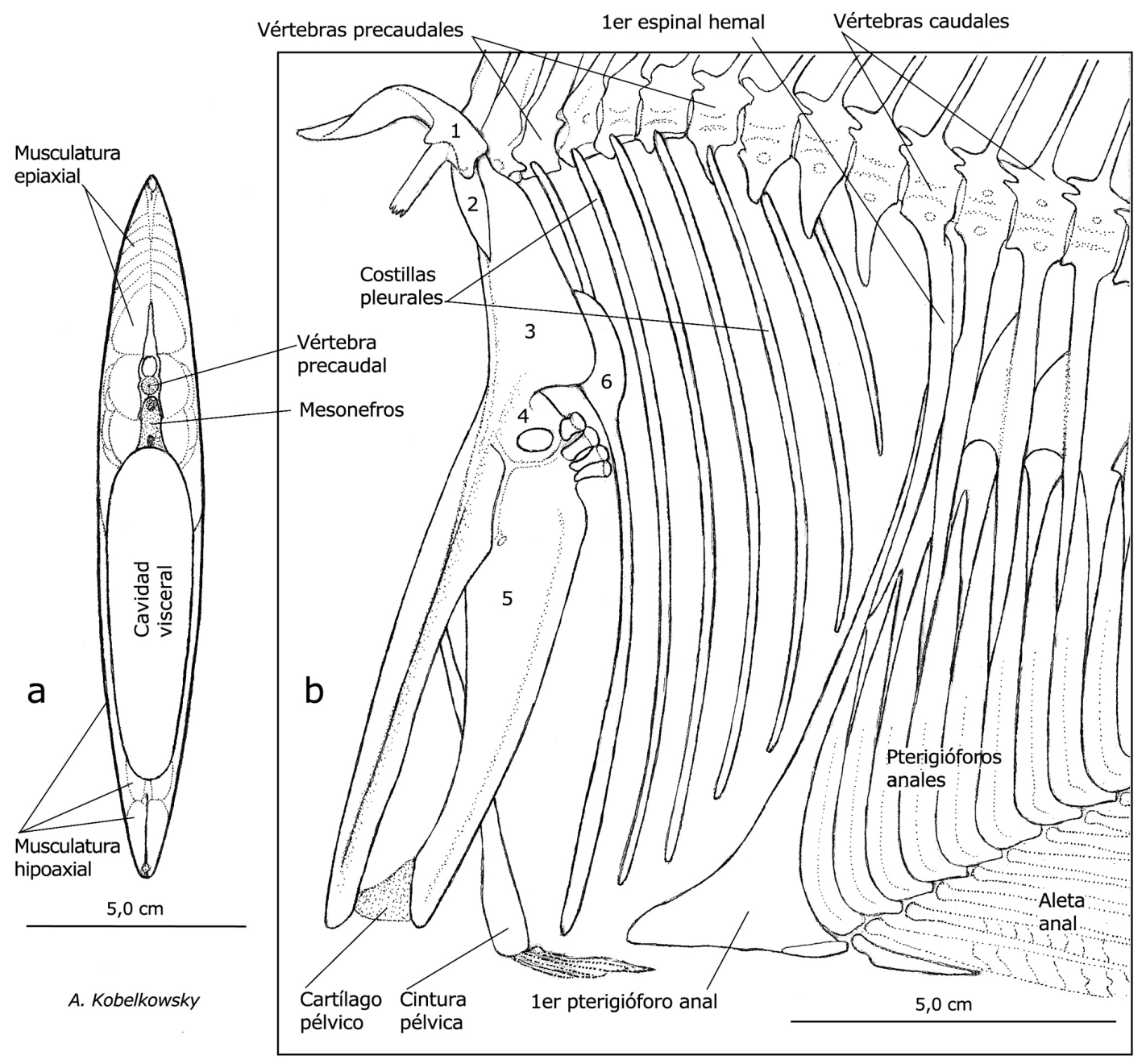

Figura 2. Límites de la cavidad visceral de Selene vomer. a) Corte transversal del cuerpo al nivel de la vértebra 7. b) Vista lateral izquierda de los límites esqueléticos de la cavidad visceral. 1, Postemporal. 2, Supracleitrum. 3, Cleitrum. 4, Escápula. 5, Coracoides. 6, Postcleitrum / Limits of the visceral cavity of Selene vomer. a) Cross section of the body at the level of vertebra 7 . b) Left lateral view of the skeletal boundaries of the visceral cavity. 1, Postemporal. 2, Supracleithrum. 3, Cleithrum. 4, Scapula. 5, Coracoid. 6, Postcleithrum

La organografía o distribución de los órganos dentro de esta cavidad celómica es en un orden descendiente el siguiente (Fig. 3): el mesonefros con carácter extraperitoneal, la vejiga gaseosa, el tubo digestivo con el hígado y el páncreas, las gónadas; el bazo se localiza entre algunas asas intestinales. El órgano que muestra el mayor volumen es la vejiga gaseosa, ocupando la porción postero-superior, mientras que el tubo digestivo, junto con sus glándulas anexas ocupan la porción antero-inferior; las gónadas se ubican con una posición oblicua, cerca del extremo inferior del primer pterigióforo anal.

\section{SISTEMA UROGENITAL}

El riñón es alargado (Figs. 3 y 4) y resulta principalmente de la fusión de los mesonefros izquierdo y derecho (Fig. 4b) desde el nivel de la vértebra 3. El inicio de sus porciones izquierda y derecha es al nivel de los huesos basioccipital y exoccipitales y se denominan lóbulos renales (Fig. 4a, b, c). Estos lóbulos se conectan con los extremos laterales del seno venoso del corazón (Fig. 4b, c).

En la vista ventral de los lóbulos renales y al nivel de las dos primeras vértebras se observan dos concavidades (Fig. $4 \mathrm{c})$, donde se localizan los músculos retractores dorsales, los cuales rodean a los dos ligamentos que suspenden a la vejiga gaseosa desde el centro vertebral 2 (Fig. 4b).

Los conductos arquinéfricos se observan cerca de los bordes inferiores del último tercio del mesonefros; se continúan hacia atrás hasta el extremo postero-inferior del órgano, de donde se desprenden y descienden en contacto uno con el otro, por el borde anterior del complejo primera espina hemal/primer pterigióforo anal, adosados a la vejiga aérea y en posición anterior al primer pterigióforo anal (Fig. 3a). Desde su extremo inferior ambos conductos se conectan a la vejiga urinaria, la cual continúa adosada al 


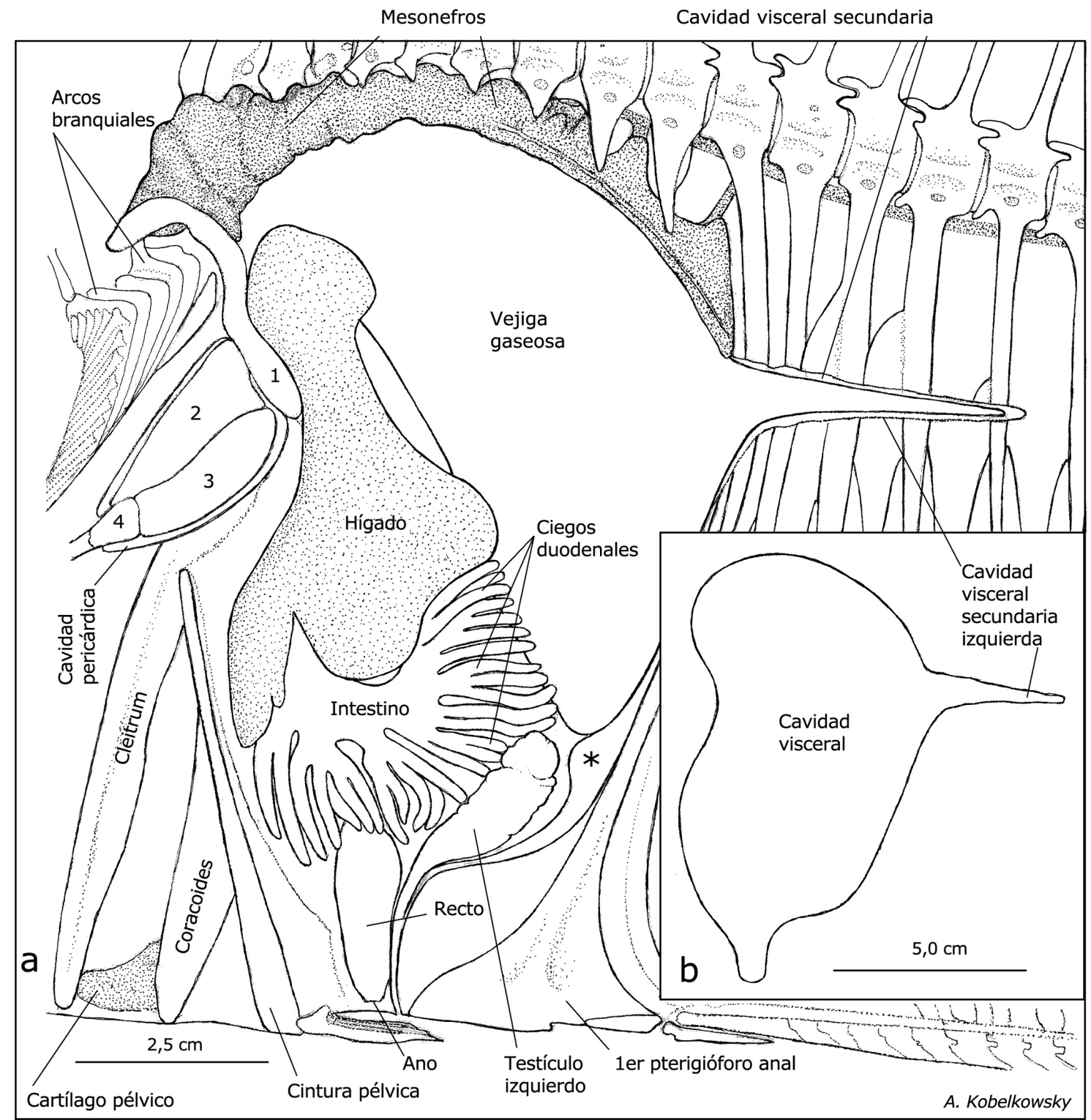

Figura 3. Organografía de las cavidades celómicas de Selene vomer. a) Vista lateral izquierda de las cavidades pericárdica y visceral y su organografía. El asterisco indica la vejiga urinaria. 1, Seno venoso. 2, Aurícula, 3, Ventrículo, 4, Cono arterial. b) Forma de la cavidad visceral en vista lateral izquierda / Organography of the celomic cavities of Selene vomer. a) Left lateral view of the pericardic and visceral cavities and their organography. The asterisk indicates the urinary bladder. 1, Sinus venosus. 2, Atrium. 3, Ventricle. 4, Conus arteriosus. b) Shape of the visceral cavity in the left lateral view

extremo inferior de la vejiga aérea. De la vejiga urinaria sale el conducto urinario común y se orienta hacia abajo y adelante. En los machos este conducto se une con el conducto espermático, formando el conducto urogenital (Fig. 4a).

La vena caudal se ubica dentro de los arcos hemales de las vértebras caudales, continuándose hacia delante hasta el arco hemal de la vértebra precaudal número ocho. Dicha vena se ramifica e introduce al mesonefros desde los arcos hemales de las vértebras precaudales nueve y diez, y desde el primer arco hemal caudal (Fig. 3a).
En la superficie ventral del riñón (Fig. 4b), se observa la vena cardinal posterior derecha, la cual se continua hasta alcanzar la porción derecha del seno venoso (Fig. 4b, c).

Los ovarios y los testículos son órganos pareados (Figs. 3a y 4a), que se localizan cerca del extremo inferior la vejiga gaseosa. Los ovarios al madurar aumentan considerablemente su volumen hacia arriba. Asimismo, los testículos aumentan su volumen hacia arriba de manera moderada. 


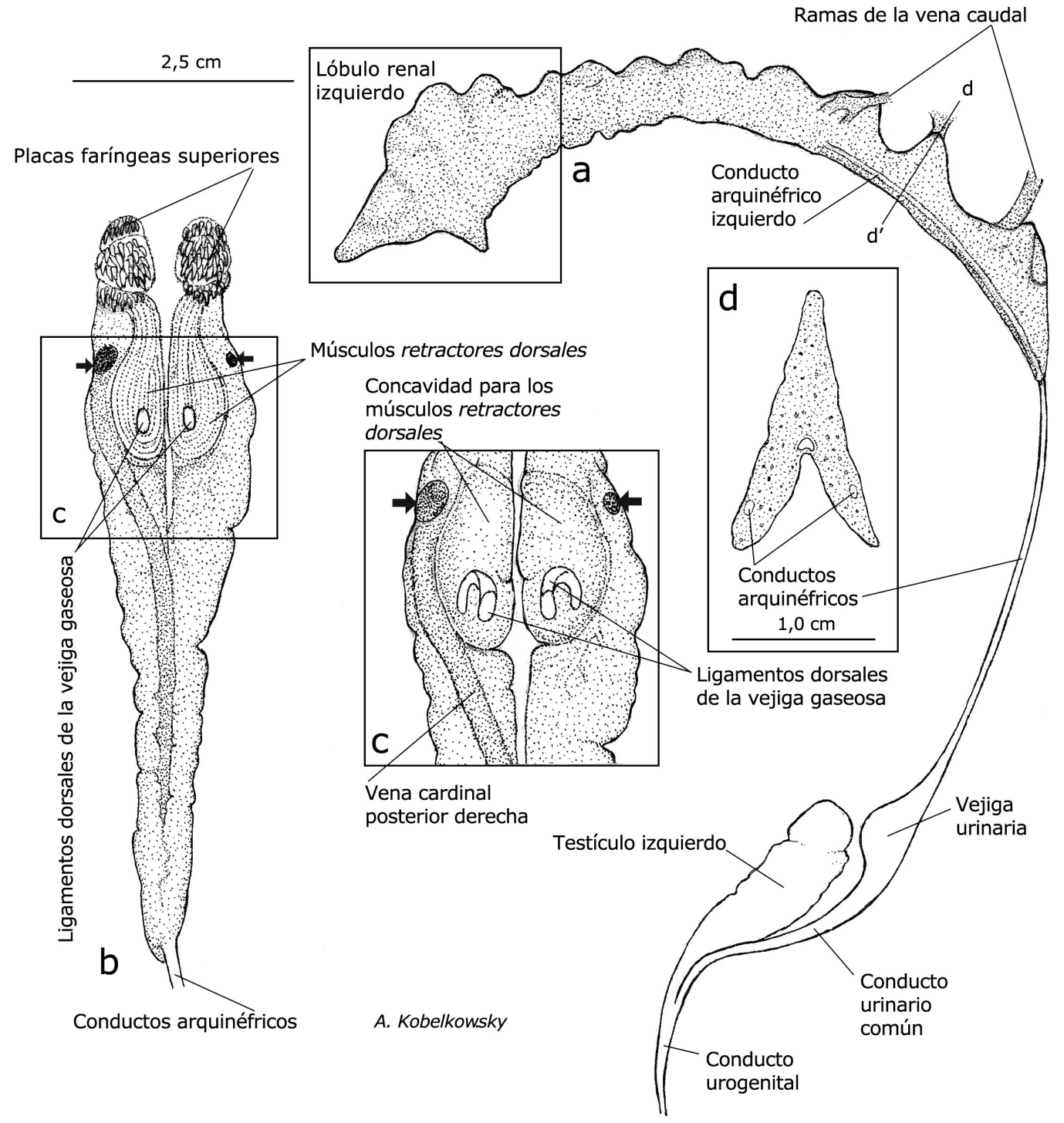

Figura 4. Sistema urogenital de Selene vomer. a) Vista lateral. b) Vista ventral del mesonefros con los músculos retractores dorsales y los huesos faríngeos superiores. c) Vista ventral de la porción anterior del mesonefros mostrando las concavidades de los músculos retractores dorsales y los ligamentos que suspenden a la vejiga gaseosa. $d$ ) Corte transversal ( $d-d^{\prime}$ ') del mesonefros realizado entre las vértebras 9 y 10 . Las flechas señalan los contactos con el seno venoso del corazón / Urogenital system of Selene vomer. a) Lateral view. b) Ventral view of mesonephros with the retractores dorsales muscles and the upper pharyngeal bones. c) Ventral view of the anterior portion of mesonephros showing the concavities of the retractores dorsales muscles and the ligaments that hold of the gas bladder. $d$ ) Cross section ( $d$ - $d^{\prime}$ ) of the mesonephros done between vertebrae 9 and 10 . Arrows point to the contacts with the sinus venosus of heart

\section{VeJIGA GASEOSA}

La vejiga gaseosa es amplia y está aplanada en el sentido lateral (Fig. 5a, b). Su aspecto lateral es semicircular y se bifurca hacia atrás en dos lóbulos posteriores delgados, que se ubican en ambos lados dentro de las "cavidades viscerales secundarias" (Fig. 3a). El anclaje de este órgano es mediante dos ligamentos dorsales, curvos, al centro vertebral 2 (Fig. 5c, d).

\section{MÚSCULOS RETRACTORES DORSALES}

Formando parte de los límites antero-dorsales de la cavidad visceral se encuentran los músculos retractores dorsales, que mueven hacia atrás los huesos faringobranquiales, y que se originan en el centro vertebral 2 (Fig. 5c). El extremo proximal de tales músculos imprime unas fosas en la parte ventral de los dos lóbulos renales y rodean a los dos ligamentos dorsales de la vejiga (Fig. 4b). 


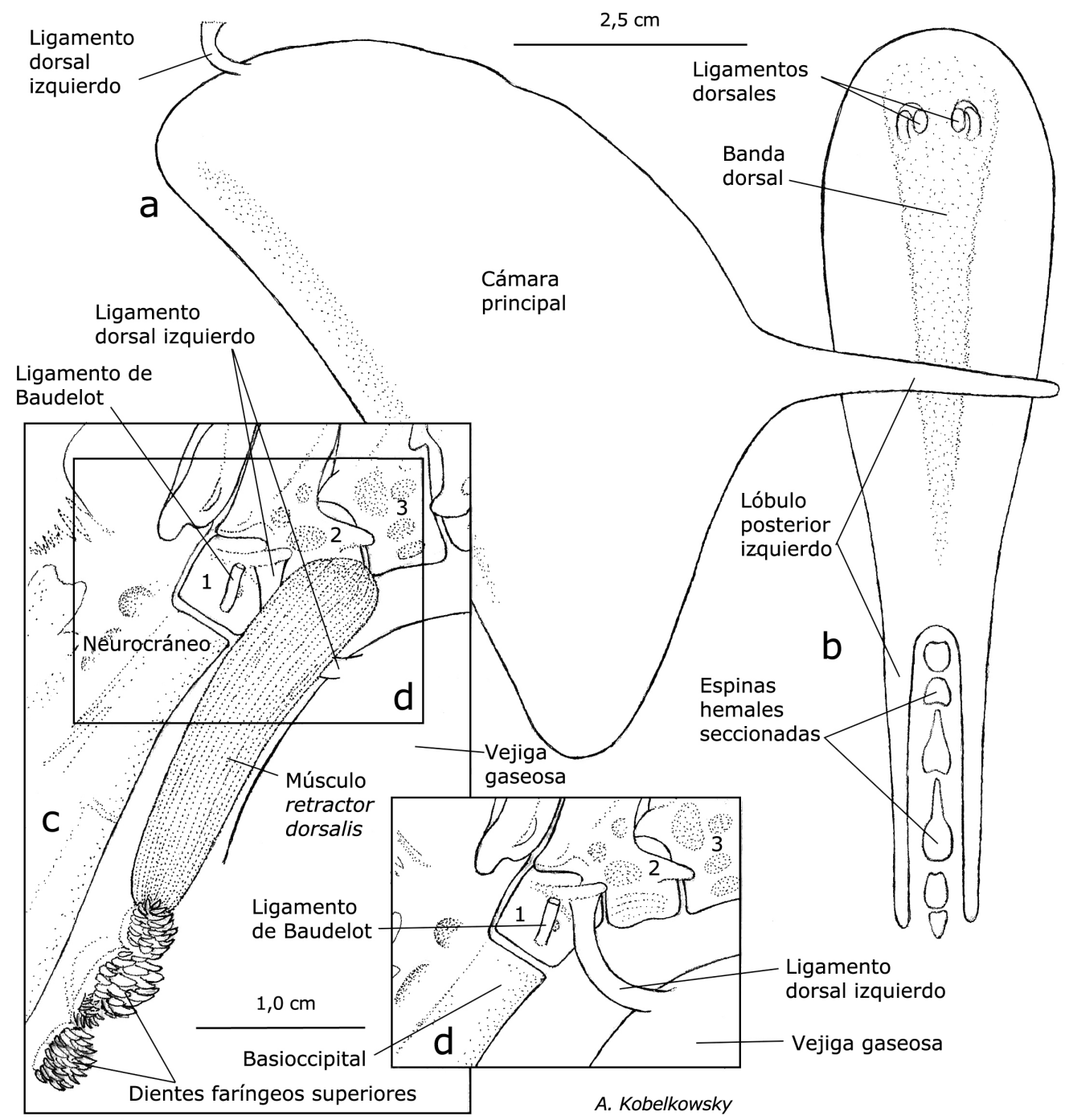

Figura 5. Vejiga gaseosa de Selene vomer. a) Vista lateral izquierda. b) Vista dorsal in situ. c) Vista lateral izquierda del músculo retractor dorsalis y el ligamento de Baudelot. d) Vista lateral izquierda del anclaje de la vejiga gaseosa al retirar el músculo retractor dorsalis izquierdo / Gas bladder of Selene vomer. a) Left lateral view. b) In situ dorsal view. c) Left lateral view of the left retractor dorsalis muscle and the left ligament of Baudelot. d) Left lateral view of the suspension of the gas bladder after removing the left retractor dorsalis muscle

\section{TUBo DIGESTIVO Y GLÁNDULAS ANEXAS}

El tubo digestivo (Fig. 6) se encuentra regionalizado de arriba hacia abajo en esófago, estómago, ciegos duodenales e intestino. El esófago es angosto y notablemente corto, y se inicia inmediatamente detrás de los huesos faríngeos superiores e inferiores, pasa entre las dos porciones del seno venoso del corazón y su pared interna muestra surcos medianos.

El estómago es relativamente largo y está regionalizado en porción cardiaca, porción pilórica y el ciego estomacal (Fig. 6d). La porción cardiaca es relativamente larga, mientras que el ciego estomacal y la porción pilórica son cortos. Su superficie interna muestra notables surcos largos y paralelos en la porción cardiaca, que en la porción pilórica son más finos. Entre el estómago y el intestino se forma el esfínter pilórico.
El intestino forma varias asas; en el aspecto lateral izquierdo (Fig. 6b) se reconoce primeramente un asa amplia, semicircular, y a continuación, en el aspecto derecho (Fig. 6c), otras tres más cortas con trayecto en "zigzag". El recto es de orientación vertical y el ano se abre en el borde medio-ventral de la cavidad visceral, entre la cintura pélvica y el primer pterigióforo anal (Fig. 3a); en la vista lateral del pez se localiza cerca de las aletas pélvicas. La longitud intestinal corresponde a 1,59 de la longitud patrón del pez.

Desde el nivel del duodeno (Fig. 6a, b), se desarrollan los ciegos duodenales en número de nueve, los cuales se ramifican de manera dicotómica y tricotómica hasta completar 72 ramas terminales; su diámetro promedio es de $0,45 \mathrm{~mm}$ y su longitud promedio es de $9,7 \mathrm{~mm}$, variando entre $9-12 \mathrm{~mm}$. 


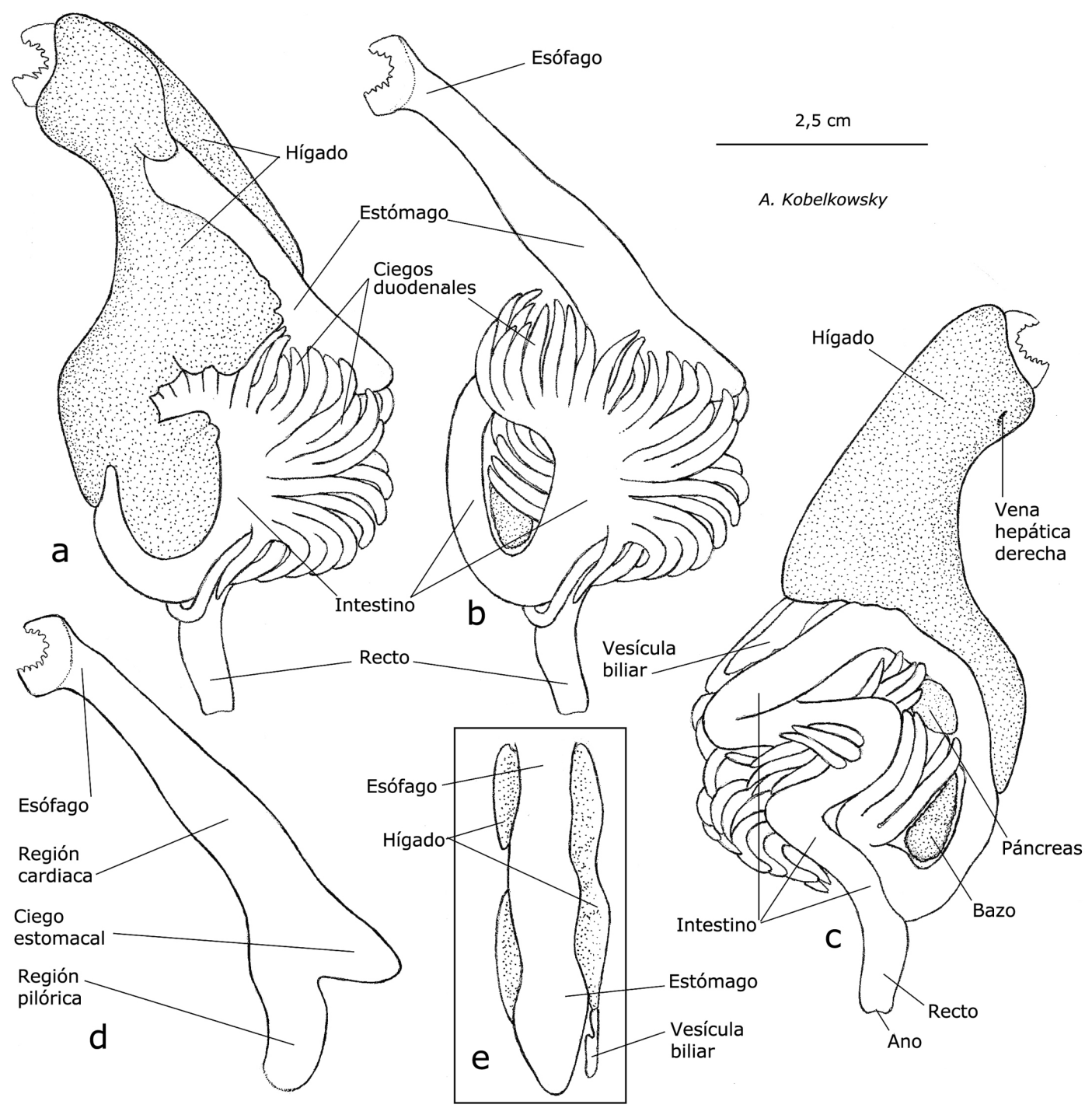

Figura 6. Tubo digestivo, hígado, páncreas y bazo de Selene vomer. a) Vista lateral izquierda del tubo digestivo y el hígado. b) Vista lateral izquierda del tubo digestivo sin el hígado. c) Vista lateral derecha del tubo digestivo y las glándulas anexas. d) Vista lateral izquierda del estómago. e) Vista dorsal del estómago y el hígado / Digestive tube, liver, pancreas, and spleen of Selene vomer. a) Left lateral view of the digestive tube and liver. b) Left lateral view of the digestive tube without the liver. c) Right lateral view of the digestive tube and annexed glands. d) Left lateral view of the stomach. e) Dorsal view of the stomach and liver

El hígado (Fig. 6a, c) es de orientación vertical, cubre ampliamente al esófago y al estómago y presenta en su borde anterior una amplia concavidad que coincide con la cavidad pericárdica (Fig. 3a). Su porción izquierda es de aspecto irregular, sin embargo, se definen un lóbulo superior izquierdo pequeño y otros dos inferiores de tamaño mediano; la porción derecha conforma un lóbulo amplio y triangular. La superficie anterior del hígado muestra las dos venas hepáticas, que se conectan al seno venoso del corazón (Fig. 6c). La vesícula biliar sobresale del ángulo posterior del lóbulo hepático derecho y tiene forma tubular.

El páncreas es ovoide y se localiza en el lado derecho, entre varios ciegos duodenales y el asa intestinal anterior (Fig. 6c).

El bazo es piriforme (Fig. 6c), de aspecto oscuro y se localiza en el lado derecho de la de masa intestinal.

\section{ASPECTOS COMPARATIVOS}

El análisis anatómico de la cavidad visceral y su organografía de las especies S. setapinnis y S. brownii muestra el mismo patrón estructural que el de $S$. vomer.

Asimismo, la definición de la forma de la cavidad visceral de especies representativas de cinco géneros de carángidos del Golfo de México, también permite reconocer el mismo patrón morfológico general de esta cavidad celómica.

Al comparar la forma de la cavidad visceral de las especies representantes de géneros de Carangidae de distribución en el Golfo de México (Fig. 7), se observa que la mayor longitud relativa de dicha cavidad es en D. punctatus; en segundo término, la de O. saurus; la menor longitud es en $S$. vomer; y la longitud intermedia se encuentra en el grupo de C. latus, H. amblyrhynchus y $C$. chrysurus. 


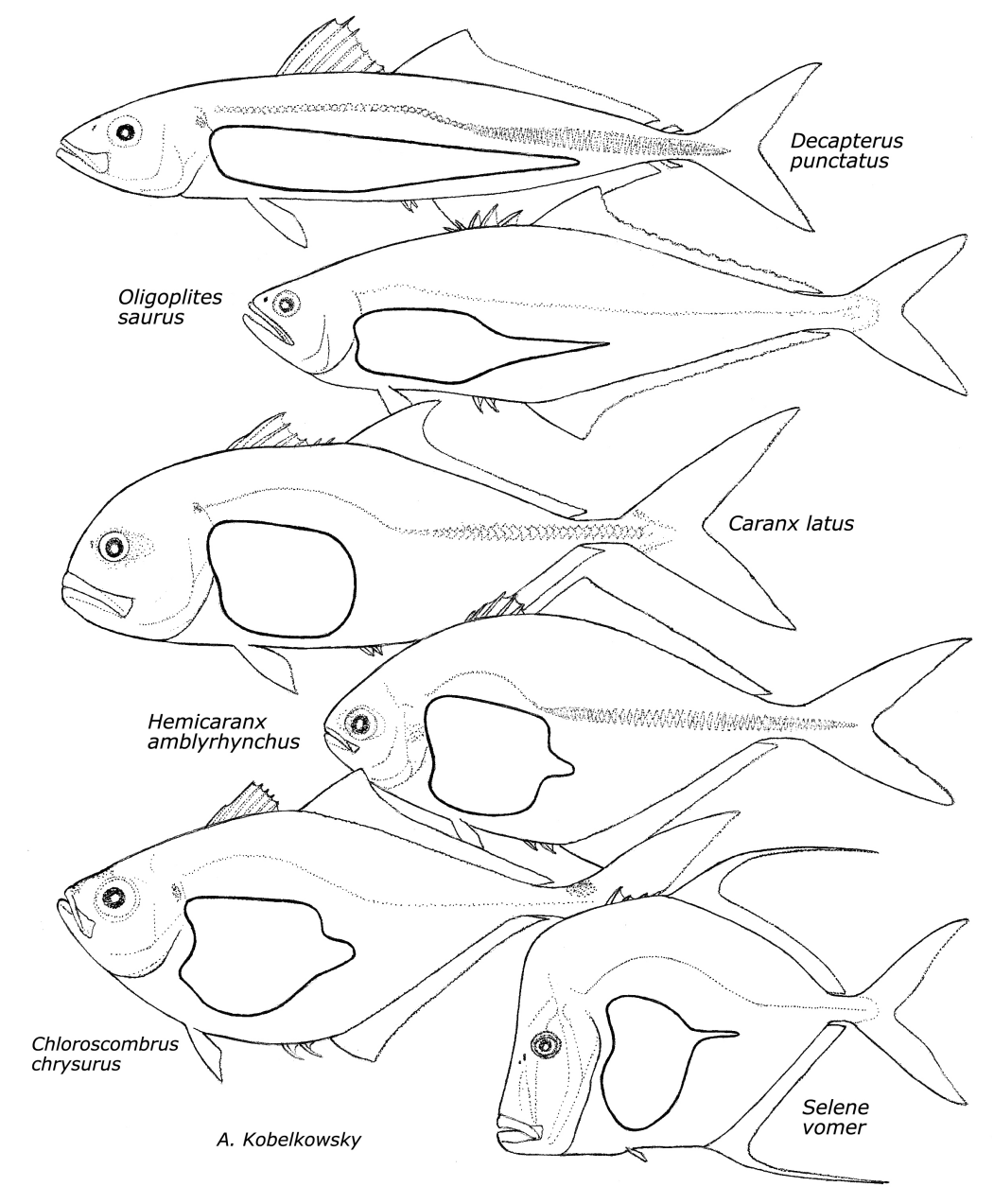

Figura 7. Comparación de la forma de la cavidad visceral en vista lateral izquierda, de una serie gradual de formas del cuerpo de especies de Carangidae / Comparison of the shape of the visceral cavity, in left lateral view, of a gradual series of body shapes of Carangidae species

Asimismo, al comparar la longitud de las “cavidades viscerales secundarias" de estas especies se reconoce aproximadamente la misma secuencia, con excepción de $C$. latus en la que no se desarrollan estas extensiones, sin embargo, la vejiga gaseosa se continúa dentro de los primeros arcos hemales.

\section{DiscuSIÓN}

Como resultado del análisis morfológico de la cavidad visceral de $S$. vomer y de los órganos que contiene, y de su comparación con $S$. setapinnis y $S$. spixi, se define un patrón anatómico del género Selene. Asimismo, al comparar la forma, particularmente de la cavidad visceral de seis especies de Carangidae del Golfo de México, con diferente forma corporal, se determinó como carácter frecuente la prolongación extra visceral de dicha cavidad celómica en las "cavidades viscerales secundarias" y la ubicación dentro de las mismas de los lóbulos posteriores de la vejiga gaseosa. Sin embargo, en C. latus no se desarrollan las cavidades viscerales secundarias, y la vejiga gaseosa se prolonga por los arcos hemales caudales.
Comparativamente con otros teleósteos es sobresaliente en las especies de Selene el alargamiento vertical de los huesos que delimitan la cavidad visceral, tales como las costillas pleurales, los cleitra, coracoides, postcleitra, cintura pélvica y el primer pterigióforo anal.

Se reconoce una convergencia anatómica de la formación de las "cavidades viscerales secundarias" de $S$. vomer con las que en los lenguados (orden Pleuronectiformes) Norman (1934) nombra como "cavidades corporales secundarias", que describe como los espacios que ocupan los ovarios maduros, y que Kobelkowsky (2007) ilustra en Cyclopsetta chittendeni. Asimismo, Norman (1934) registra, en los lenguados de la familia Achiridae la presencia de dos asas intestinales en la "cavidad visceral secundaria derecha", acompañando al ovario derecho. Kobelkowsky (2000) describe e ilustra dicha condición en tres especies de dicha familia reconociendo, además, en esa misma ubicación al lóbulo renal posterior y a la vejiga urinaria. 
La anatomía gruesa del riñón de $S$. vomer es similar a la de otros teleósteos como la que describe Kobelkowsky (2013) en Lutjanus griseus (Lutjanidae).

Relacionado directamente con la reducida longitud de la cavidad visceral y el "adelantamiento" del primer pterigióforo anal, se encuentra la orientación oblicua de las gónadas en ambos sexos. Esto implica que los conductos reproductores están ligeramente orientados hacia delante, situación relativamente similar a la del lenguado Citharichthys spilopterus descrita por Kobelkowsky \& Castillo-Rivera (2017).

Entre los órganos de la cavidad visceral, los que muestran características especiales son el tubo digestivo y la vejiga gaseosa.

La mayoría de los teleósteos que presentan ciegos pilóricos o ciegos duodenales, estos son de carácter simple, mientras que en $S$. vomer son ramificados, de manera similar a los que describen Kobelkowsky \& Pérez-García (2007) en Elops saurus (Elopidae). La relación longitud intestinal/longitud patrón del pez en $S$. vomer indica un hábito carnívoro de alimentación.

Los dos caracteres sobresalientes de la vejiga gaseosa de $S$. vomer son: a) los ligamentos que la suspenden desde la columna vertebral, situación no registrada en la literatura, y b) el desarrollo extra-visceral de los dos lóbulos posteriores, de manera similar a lo descrito por Terán-Martínez (2015) en Gerres cinereus (Gerreidae).

\section{LITERATURA CITADA}

Collette BB \& LN Chao. 1975. Systematics and morphology of the bonitos (Sarda) and their relatives (Scombridae, Sardini). Fishery Bulletin 73(3): 516-625.

Gregory WK. 1959. Fish skull. A study of evolution of natural mechanisms, 481 pp. Eric Lundberg, Laurel.

Guzmán AF \& OJ Polaco. 2002. Hiperostosis en peces mexicanos. Archaeofauna 11: 45-62.

Harder W. 1958. El intestino como carácter diagnóstico para la identificación de ciertos clupeioides (Engraulidae, Clupeidae, Dussumieriidae) y como carácter morfométrico para la comparación de las poblaciones de anchoveta (Cetengraulis mysticetus). Comisión Interamericana del Atún Tropical, Boletín 8: 381-376.

Kobelkowsky DA. 2000. Sistema urogenital de los lenguados de la familia Achiridae (Pisces: Pleuronectiformes) del Golfo de México. Hidrobiológica 10(1): 51-60.
Kobelkowsky A. 2007. Morphology of the digestive system of the Mexican flounder, Cyclopsetta chittendeni (Teleostei, Paralichthyidae). Bulletin of Fish Biology 9: 39-49.

Kobelkowsky A. 2012. Morphological diversity of the ovaries of the Mexican teleost fishes. International Journal of Morphology 30(4): 1353-1362.

Kobelkowsky A. 2013. Morphology and dissection technique of the kidney of the grey snapper Lutjanus griseus (Teleostei: Lutjanidae). International Journal of Morphology 31(2): 553-561.

Kobelkowsky A \& MA Pérez-García. 2007. El sistema digestivo de Elops saurus (Teleostei: Elopidae). Vertebrata Mexicana 20: 1-7.

Kobelkowsky A \& MA Castillo-Rivera. 2017. Sexual anatomy of the flounder Citharichthys spilopterus (Paralichthyidae). Cybium. International Journal of Ichthyology 42(2): 129136.

Nelson JS, TC Grande \& MVH Wilson. 2016. Fishes of the world, 711 pp. John Wiley \& Sons, Hoboken.

Norman JR. 1934. A systematic monograph of the flatfishes (Heterostomata). Vol. 1, Psettodidae, Bothidae, Pleuronectidae, $480 \mathrm{pp}$. British Museum of Natural History, London.

Ogawa M. 1961. Comparative study of the external shape of the teleostean kidney with relation to phylogeny. Science Reports of the Tokyo Kyoiku Daigaku, B10: 61-68.

Robins CR \& GC Ray. 1986. A field guide to Atlantic coast fishes of North America, 354 pp. Houghton Mifflin Company, Boston.

Sasaki K. 1989. Phylogeny of the family Sciaenidae, with notes on its geography (Teleostei, Perciformes). Memoirs of the Faculty of Fisheries Hokkaido University 36(1-2): 1-37.

Terán-Martínez J. 2015. Diversidad morfológica de la vejiga gaseosa en la familia Gerreidae (Pisces: Teleostei). Tesis de Maestría en Ciencias, Ciencias Marinas, Posgrado de Ciencias del Mar y Limnología, Universidad Nacional Autónoma de México, Ciudad de México, 86 pp.

Whitehead PJP \& JHS Blaxter. 1989. Swimbladder form in clupeoid fishes. Zoological Journal of the Linnean Society 97(4): 299-372.

Wilson JM \& LFC Castro. 2011. Morphological diversity of the gastrointestinal tract in fishes. In: Grosell M, AP Farrell \& CJ Brauer (eds). The multifunctional gut of fish, pp. 2-55. Academic Press, London.

Winterbottom RA. 1974. A descriptive synonymy of the striated muscles of the Teleostei. Proceedings of the Academy of Natural Sciences of Philadelphia 125(12): 225-317. 